\title{
Using Image Similarity and Asymmetry to Detect Breast Cancer
}

\author{
Dave Tahmoush and Hanan Samet \\ Computer Science Department, University of Maryland, College Park;
}

\begin{abstract}
Radiologists can use the differences between the left and right breasts, or asymmetry, in mammograms to help detect certain malignant breast cancers. An image similarity method is introduced to make use of this knowledge base to recognize breast cancer. Image similarity is determined using a contextual and then a spatial comparison. The mammograms are filtered to find the most contextually significant points, and then the resulting point set is analyzed for spatial similarity. We develop the analysis through a combination of modeling and supervised learning of model parameters. This process correctly classifies mammograms $80 \%$ of the time and thus asymmetry is a measure that can play an important role in significantly improving computer-aided breast cancer detection systems.
\end{abstract}

Keywords: Computer-Aided Detection, asymmetry, image similarity, breast cancer, prescreening

\section{INTRODUCTION}

Breast cancer remains a leading cause of cancer deaths among women in many parts of the world. In the United States alone, over forty thousand women die of the disease each year. ${ }^{1}$ Mammography is currently the most effective method for early detection of breast cancer. ${ }^{2}$ For two-thirds of the women whose initial diagnosis of their mammogram is negative but who actually have breast cancer, the cancer is evident upon a second diagnosis of their mammogram. ${ }^{2}$ Computer-aided detection (CAD) of mammograms could be used to avoid these missed diagnoses, and has been shown to increase the number of cancers detected by more than nineteen percent. ${ }^{3}$ Measuring asymmetry, which consists of a comparison of the left and right breast images, ${ }^{4}$ is a technique that could be used to improve the accuracy of CAD. An automated prescreening system only classifies a mammogram as either normal or suspicious, while CAD picks out specific points as cancerous. ${ }^{5}$ One of the most challenging problems with prescreening is the lack of sensitive algorithms for the detection of asymmetry. ${ }^{6}$ This paper presents a simple and effective algorithm for the detection of asymmetry and extensions to improve upon it.

Mammograms are an excellent candidate for image similarity techniques to be effective because there are images of both the left and right breasts, which should be similar if there is no cancer present. Image similarity has been often utilized for content-based image retrieval (CBIR) from image databases ${ }^{789} .{ }^{10}$ Both contextual and spatial comparisons are used. ${ }^{7}$ Medical image databases have also used image similarity, from rule-based systems for chest radiographs ${ }^{11}$ to anatomical structure matching for 3D MR images. ${ }^{12}$ However, the focus is often on the non-cancerous structures, while it is the cancerous structures that are of principle interest here. In this paper we combine the image similarity concept of contextual then spatial comparison to the problem of detecting breast cancer in mammograms.

The majority of work on CAD analysis of mammograms has focused on determining the contextual similarity to cancer, finding abnormalities in a local area of a single image ${ }^{13} \cdot{ }^{14}$ This paper focuses on combining this with a spatial comparison in order to complete an image similarity measure. The majority of work has used methods ranging from filters to wavelets to learning techniques, but a detailed discussion of various imaging techniques is beyond the scope of this paper. Problems arise in using filter methods ${ }^{13}$ because of the range of sizes and morphologies for breast cancer, as well as the difficulty in differentiating cancerous from non-cancerous structures. The size range problem has been addressed by using multi-scale models. ${ }^{14}$ Similar issues affect wavelet methods, although their use has led to reported good results ${ }^{15}$ with the size range issue being improved

E-mail: tahmoush@cs.umd.edu,hjs@umiacs.umd.edu

Medical Imaging 2006: Image Processing, edited by Joseph M. Reinhardt, Josien P. W. Pluim, Proc. of SPIE Vol. 6144, 61441S, (2006) · 0277-786X/06/\$15 - doi: 10.1117/12.652643 
through the use of a wavelet pyramid. ${ }^{16}$ Learning techniques have included support vector machines ${ }^{17}$ and neural networks. ${ }^{15}$

Detecting breast cancer is challenging because the cancerous structures have many features in common with normal breast tissue. This means that a high number of false positives or false negatives are possible. Asymmetry can be used to help reduce the number of false positives so that true positives are more obvious. Previous work utilizing asymmetry has used wavelets or structural clues to detect asymmetry with correct results as often as $77 \%$ of the time ${ }^{4} .^{18}$ Additional work has focused on bilateral or temporal subtraction, which is the attempt to subtract one breast image from the other ${ }^{19} .^{20}$ This approach is good because it does try to utilize the multiple images taken with the same machine by the same technician and analyzed using the same process in an effort to reduce the systematic differences that can be introduced. However, bilateral subtraction is hampered by the necessity of exact registration and natural asymmetry of the breasts. We introduce a measure of asymmetry that is more approximate in nature and seems more robust to the large amount of noise in the data, using learning to determine a highly constrained number of model parameters. Minimizing the number of parameters that are learned makes the model less subject to the noise in the data at the expense of accuracy.

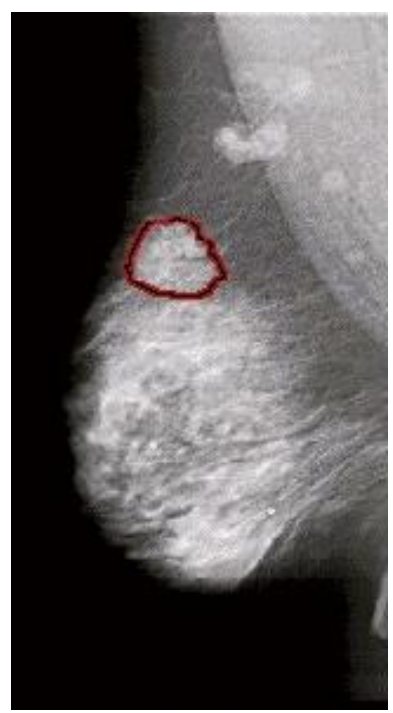

(a)

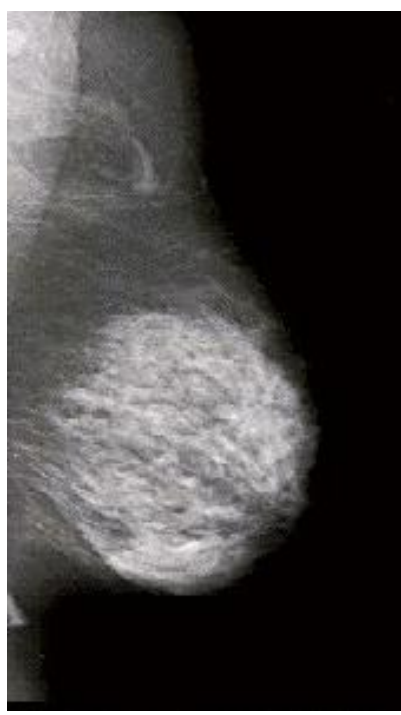

(b)

Figure 1: Mammograms of left and right breasts with cancerous area outlined. The similarity of texture between cancerous and normal tissue makes asymmetry an important tool in cancer detection.

Comparing multiple mammograms using learning techniques has been shown to be effective in $\mathrm{CBIR}^{9} .^{21}$ Neural nets can be an effective supervised learning technique. ${ }^{22}$ Our application lends itself well to supervised learning because the data set has already been screened for cancer and thus classified by expert radiologists. However, care must be taken since the expert classification is known not to be perfect. ${ }^{2}$ A difficulty in utilizing learning techniques is creation of an accurate model to learn. ${ }^{22}$

We believe that developing ways to better utilize asymmetry is consistent with a philosophy of trying to use methods that can capture measures deemed important by doctors thereby building upon their knowledge base, instead of trying to supplant it. However, measuring asymmetry means comparing multiple images, and thus it is a more complicated process.

The rest of this paper is organized as follows. Section 2 presents our method for measuring asymmetry between the mammogram images. Section 3 discusses the evaluation of the performance of the measure. Section 4 compares the results with other work, Section 5 describes future work, while Section 6 discusses the conclusions that can be drawn from this work. 


\section{ASYMMETRY MEASUREMENT}

Our work utilizes filtering followed by spatial analysis to determine an overall measure of similarity by combining the contextual similarity of the filtering with the spatial similarity of the analysis. This can be a useful measure for prescreening mammograms since only an overall determination is required. We believe that many of the techniques described here can also be adapted for use in CAD analysis. A secondary goal of our work is to determine the importance of similarity or asymmetry in the computer analysis of mammograms. Figure 1 shows why spatial asymmetry is important in finding cancers in mammograms since we see that the texture and appearance of cancer are both very similar to the texture and appearance of normal tissue in the breast.

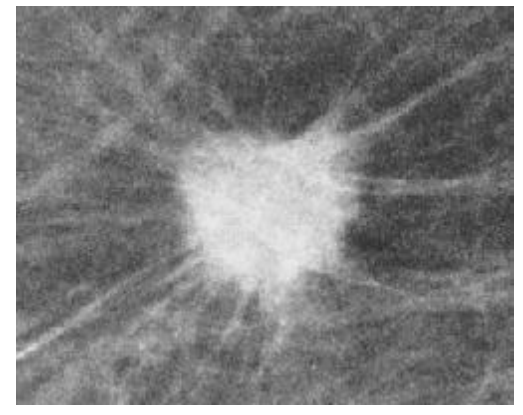

(a)

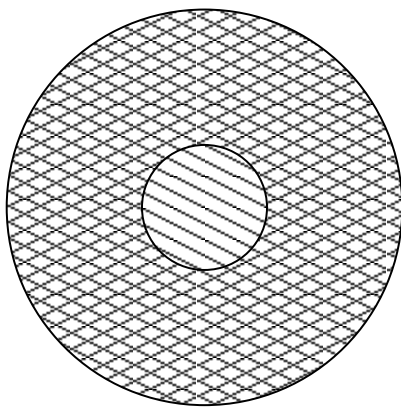

(b)

Figure 2: A mammographic image of a spiculated lesion is in (a). The bright center or core is one feature of these lesions, as well as the radiating lines which are called spiculations. A relative brightness detection filter is in (b). The filter calculates the percent of the pixels in the outer ring that are less bright than the least bright of the pixels in the inner disk. The filter highlights the areas of the image that are brighter than their surroundings, like the bright central core of spiculated lesions.

Our analysis starts with filtering to find the contextually similar suspicious points that could be cancers in the mammograms. The filter calculates the percent of the pixels in the outer ring that are less bright than the least bright of the pixels in the inner disk to produce a suspiciousness value, and an example is given in Figure $2 \mathrm{~b}$. This suspiciousness value represents the degree to which the surrounding region of a point radially decreases in intensity. This filter is designed to pick out the bright central core of spiculated lesions. Points with a high suspiciousness value have a higher chance of corresponding to an occurrence of cancer. The centroid of each local maxima in the filtered image is initially marked as a candidate detection site with its suspiciousness value. This collection of sites is then sorted in decreasing order of suspicion. All suspicious sites that are closer than $5 \mathrm{~mm}$ from a more suspicious site are removed to prevent multiple reporting of the same site. This yields a set of potential detection sites that can be analyzed for asymmetry. This technique was advanced by Heath. ${ }^{23}$ Although it may not be the optimal choice of either filtering or ranking, the spatial analysis that we used can be applied to any technique that can rank the suspiciousness of areas. We limit the analysis to the top thirty-two suspicious points. The number of points returned by the filtering step is one of the variables that can be adjusted to optimize the analysis. Alternatively, we can also make use of a threshold on the suspiciousness value instead of taking the top few. However, we chose to take the top few in order to be insensitive to image processing choices that might bias the analysis.

The analysis for similarity or asymmetry that we used does a comparison of the locations of the sets of suspicious points. A separator is learned with a training set of images. This separator breaks the set of suspicious points into two groups, and the populations in the groups are compared between the images. This is based upon the assumption that the presence of cancer will distort the distribution of suspicious sites, and that the distribution will be very similar from left to right breasts when there is no cancer. This is similar to comparing histograms whose parameters are learned from a test set. We found a natural separation of the two clusters in many of the mammograms, which can be seen in the right breast in Figure 3a. This is important in preventing noise from being introduced through small variations in the positions of the suspicious points. We 


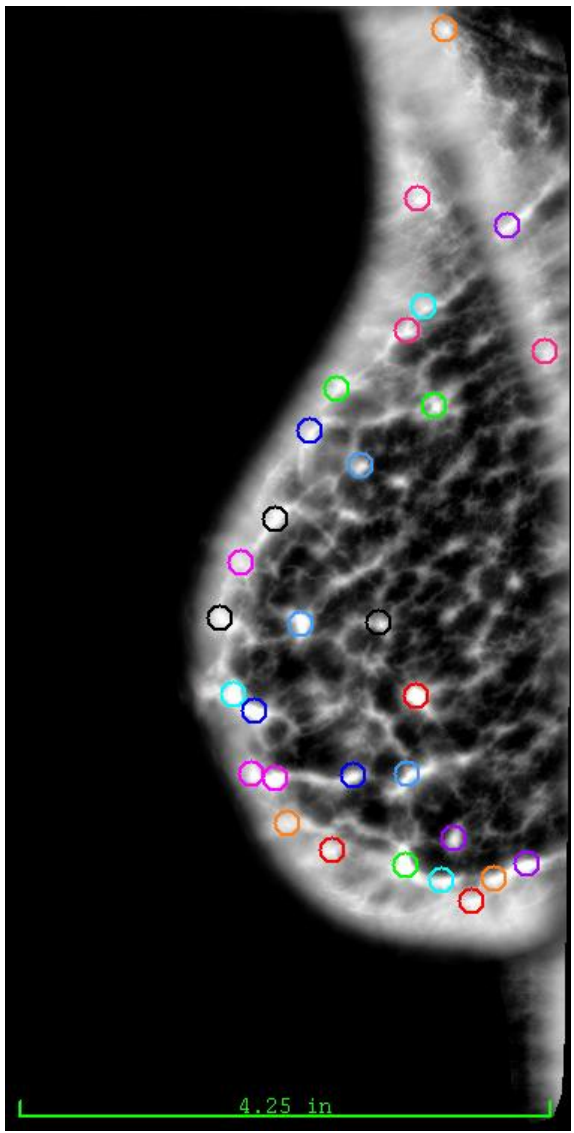

(a)

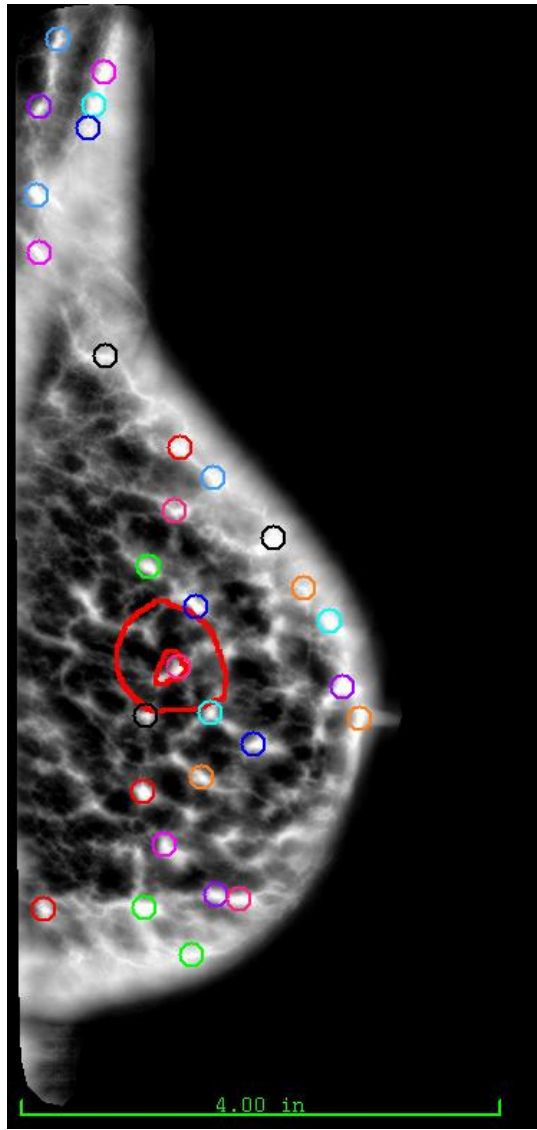

(b)

Figure 3: The two breasts are shown, with the suspicious points indicated by circles. The two hand-drawn circles (one inside the other) in the right breast are the radiologist's diagnosis of cancer. The asymmetry is demonstrated by the presence of considerably fewer suspicious points in the matching area in the left breast - that is, the distribution of suspicious points changes slightly from one breast to the other when there is cancer. Note that there are circles within the hand-drawn circles, showing that the filtering does find the cancer.

used learning techniques to determine the optimal structure and parameters for the separator from the data. For this application, the importance of correct classification of the cancerous cases is much more important than the non-cancerous cases. To reflect this, the associated weighting of the cancerous cases was varied, and we evaluate the performance of various weightings.

\section{EVALUATION}

The groups of suspicious points in the left and right mediolateral oblique (MLO) mammogram views were compared to evaluate the asymmetry measure. The analysis was done over two data sets, with cases that were split between normal mammograms and mammograms with malignant spiculated lesions from the Digital Database for Screening Mammography. ${ }^{23}$ Spiculated lesions are defined as breast cancers with central areas that are usually irregular and with ill-defined borders. Their sizes vary from a few millimeters to several centimeters in diameter and they are very difficult cancers to detect. ${ }^{16}$ The training set had 20 non-cancerous cases and 40 cancerous cases, while the test set had 25 non-cancerous cases and 37 cancerous cases. The data is roughly spread across the density of the breasts and the subtlety of the cancer. The breast density and subtlety were specified by an expert radiologist. The subtlety of the cancer shows how difficult it is to determine that there is 


\begin{tabular}{|l|r|r|r|}
\hline Method & Cancerous & Normal & Total \\
\hline Equal Weights & $87 \%$ & $71 \%$ & $80 \%$ \\
\hline Weighted Toward Cancer & $97 \%$ & $42 \%$ & $74 \%$ \\
\hline R2 ImageChecker & $96 \%$ & $33 \%$ & $70 \%$ \\
\hline Wavelet & $77 \%$ & $77 \%$ & $77 \%$ \\
\hline Naive Bayes & $66 \%$ & $33 \%$ & $52 \%$ \\
\hline
\end{tabular}

Table 1: Results Table. The results compare favorably against the R2 Imagechecker system and other techniques.

cancer. The training data set was used to determine optimal parameters for classification. These cases indicated that a difference in the groups of one or more suspicious points indicated cancer. The mammogram in Figure 3a shows how the spatial distribution of suspicious points is changed by the presence of a cancer.

\section{RESULTS}

Our results are good on all cases of the test set, correctly classifying $80 \%$ on the test set. However, it is much more important to correctly classify the cancerous cases, and by heavily weighting the importance of the cancerous cases, we correctly classify $97 \%$ of the cancerous cases and $42 \%$ of the non-cancerous cases. Neither the subtlety nor the density of the cancer had an effect on the results. The comparison with a commercial system shows that the results are surprisingly good. Correct classification results of $96 \%$ of the cancerous cases and $33 \%$ of non-cancerous cases are possible using the R2 ImageChecker system. ${ }^{6}$ Our method showed correct classification results on $97 \%$ of cancerous cases and $42 \%$ of the non-cancerous cases. This demonstrates the importance of asymmetry in pre-screening, since using only asymmetry achieves a better performance than a complete comercial system. The inclusion of additional factors other than asymmetry in the method should improve the results. However, the data sets used are different, as the R2 ImageChecker data contains all cancer types and our method has only the difficult to detect spiculated lesions. The R2 ImageChecker data set also had a much higher proportion of non-cancerous mammograms to cancerous cases.

Our method makes use of a spatial analysis of the suspicious points, counting the number of suspicious points in the groups created by the separator. Its success is an encouraging sign for the investigation and utilization of more complicated non-local analysis techniques in medical imaging and analysis.

\section{FUTURE WORK}

In the future we plan to move away from the concept of separators to clusters. In some sense, separating planes in the data space create areas that can be defined as clusters, but these clusters are restricted to be space-filling and non-overlapping. These restrictions are not required, and performance could improve with their removal.

This work used only one separator. Increasing the number of separators, or clusters, should improve the performance. Initial work has shown an improvement to $84 \%$ accuracy. Using multiple separators provides a way to probe the data space for regions of interest, either because they are important areas for finding cancer or for determining that there is no cancer. The initial analysis has discovered a small region of interest for diagnosing a mammogram as non-cancerous.

This algorithm is not expected to work well on every case because there are to many types of breast cancer and breast characteristics. Tuning the algorithm to the characteristics of the breasts should improve the results. Additionally, letting the algorithm decide when it can do well and when it cannot should also improve performance. Initial results show an improvement in accuracy to $84 \%$ by allowing the one separator model to select which cases it thinks that it can analyze.

One of the parameters that can be learned is the optimal number of suspicious points to use in the analysis. Initial results were always at or near the top of the range that we used, varying from 29 to 32 points depending on the model and weightings. This was surprising because the cancer was usually in the top sixteen if not the top eight points. However, the suspicious points do tend to cluster around a cancer, so including more suspicious 
points may create a greater distortion of the underlying distribution than fewer points. The learning algorithm does not get the number of points directly, only the cluster differences, so the inclusion of more data should not result in overfitting.

A further improvement might be possible by first transforming the data before filtering, such as applying wavelet analysis to the images before simply thresholding or applying the filter. This has been successfully attempted previously ${ }^{4}$ with good results. However, an optimal solution would first combine all of the various filtering and transform methods which create meaningful suspicious points, and then learn an effective analysis from them. This is similar to the effective combination of weak classifiers into a single strong classifier through ensemble learning methods like boosting, which has been successfully used before in tumor classification. ${ }^{24}$

\section{CONCLUSION}

The overall results of using our techniques are good, our experiments on malignant cases yielded $80 \%$ accuracy suggesting that asymmetry is an important measure to incorporate into prescreening or CAD software. The technique can be tuned to be more effective at diagnosing cancerous cases, reaching $97 \%$ accuracy. We suggest several ways to improve on the methods that we used to measure asymmetry. One method is to convert a mammogram into a connected graph structure of suspicious points and utilize known graph comparison methods for the measure. Another is to use registration of suspicious points from one breast to the other and reduce the suspiciousness of points that have a similar counterpart. Alternatively, increasing the number of separators could improve the method.

Our work has demonstrated the potential of utilizing techniques like image comparisons and other non-local methods with medical imaging. We have shown that we can effectively measure doctor-defined quantities like asymmetry. We believe that in the future, the combination of capturing doctor-defined quantities like asymmetry and machine learning of parameters could be a powerful method for improving the quality of research in medical imaging, and this is one of the avenues of research that we intend to pursue.

\section{ACKNOWLEDGMENTS}

This work was supported in part by the National Science Foundation under grants EIA-00-91474 and CCF0515241, and by Microsoft Research.

\section{REFERENCES}

1. American Cancer Society. Breast Cancer Facts and Figures 1999-2000. American Cancer Society, Inc., Atlanta, GA, 1999.

2. J. Linda, W. Burhenne, S.A. Wood, C.J. D’Orsi, S.A. Feig, D.B. Kopans, K.F. O'Shaughnessy, E.A. Sickles, L. Tabar, C.J. Vyborny, and R.A. Castellino. Potential contribution of computer-aided detection to the sensitivity of screening mammography. Radiology, 215(554-562), 2000.

3. T.W. Freer and M.J. Ulissey. Screening mammography with computer- aided detection. Radiology, 220:781$786,2001$.

4. R.J. Ferrari, R.M. Rangayyan, J.E.L. Desautels, A.F. Frere. Analysis of asymmetry in mammograms via directional filtering with Gabor wavelets. IEEE Trans. on Medical Imaging, 20(9), 2001.

5. S. Astley, T. Mistry, C.R.M. Boggis, V. F. Hillier. Should we use humans or a machine to pre-screen mammograms? In Proc. of the Sixth Int. Workshop on Digital Mammography, pp 476-480, 2002.

6. S. Astley and F.J. Gilbert. Computer-aided detection in mammography. Clinical Radiology, 59:390-9, 2004.

7. A. Soffer and H. Samet. Pictorial queries by image similarity. In Proc. of the 13th Int. Conf. on Pattern Recognition, vol. 3, pp 114-119, 1996.

8. V. Gudivada and V. Raghavan. Design and evaluation of algorithms for image retrieval by spatial similarity. ACM Trans. on Inf. Sys., 13(2):115-144, 1995.

9. I. El-Naqa, Y. Yang, N.P. Galatsanos, R.M. Nishikawa, and M.N. Wernick. A similarity learning approach to content based image retrieval: application to digital mammography. IEEE Trans. on Medical Imaging, 23(10):1233-1244, 2004. 
10. J. Goldberger, S. Gordon, and H. Greenspan. An efficient image similarity measure based on approximations of kl-divergence between two gaussian mixtures. In Proc. of the Ninth IEEE Int. Conf. on Computer Vision, pp 487-493, 2003.

11. H.A. Swett and P.L.Miller. Icon: a computer-based approach to differential diagnosis in radiology. Radiology, 163:555-558, 1987.

12. A. Guimond and G. Subsol. Automatic mri database exploration and applications. Pattern Recognition and Artificial Intelligence, 11(8):1345-1365, 1997.

13. M.D. Heath and K.W. Bowyer. Mass detection by relative image intensity. In The Proc. of the 5th Int. Conf. on Digital Mammography, Medical Physics Publishing, Madison, WI, 2000.

14. P. Sajda, C. Spense, and L. Parra. Capturing contextual dependencies in medical imagery using hierarchical multi-scale models. In Proc. of the IEEE Int. Symp. on Biomedical Imaging, pp 165-168, 2002.

15. B.L. Kalman, S.C. Kwasny, and W.R. Reinus. Diagnostic screening of digital mammograms using wavelets and neural networks to extract structure. Technical Report 98-20, Washington University, 1998.

16. S. Lui, C.F. Babbs, and E.J. Delp. Multiresolution detection of spiculated lesions in digital mammograms. IEEE Trans. on Image Processing, 6:874-884, 2001.

17. R. Campanini, A. Bazzani, A. Bevilacqua, D. Bollini, D. Dongiovanni, E. Iampieri, N. Lanconelli, A. Riccardi, M. Roffilli, and R. Tazzoli. A novel approach to mass detection in digital mammography based on support vector machines. In Proc. of the 6th Int. Workshop on Digital Mammography, 2002.

18. P. Miller and S. Astley. Detection of breast asymmetry using anatomical features. In Proc. of the Int. Society for Optical Engineering Conf. on Biomedical Image Processing and Biomedical Visualization, 1905:433-442, 1993.

19. M.A. Wirth and A. Jennings. A nonrigid-body approach to matching mammograms. In Proc. of the IEEE Image Processing and its Applications, pp 484-7, 1999.

20. F.F. Yin, M.L. Giger, K. Doi, C.E. Metz, C.J. Vyborny, and R.A. Schmidt. Computerized detection of masses in digital mammograms: analysis of bilateral subtraction images. Medical Physics, 18:955-63.

21. I. Gondra and D.R. Heisterkamp. Learning in region-based image retrieval with generalized support vector machines. In Proc. of the Computer Vision and Pattern Recognition, p 149, 2004.

22. J.A. Anderson, 1995, An Introduction to Neural Networks, The MIT Press.

23. M.D. Heath, K.W. Bowyer, D. Kopans et al. Current status of the digital database for screening mammography. Digital Mammography, Kluwer Academic Publishers, pp 457-60, 1998.

24. M. Dettling and P. Buhlmann. Boosting for tumor classification with gene expression data. Bioinformatics, 19(9)1061-1069, 2003. 